\title{
Linx
}

Revue des linguistes de l'université Paris X Nanterre

5 | 1994

La négation

\section{Pas et point en français classique}

André Meunier et Mary-Annick Morel

\section{(2) OpenEdition}

Journals

Édition électronique

URL : http://journals.openedition.org/linx/1232

DOI : 10.4000/linx.1232

ISSN : 2118-9692

\section{Éditeur}

Presses universitaires de Paris Nanterre

\section{Édition imprimée}

Date de publication : 1 juin 1994

Pagination : $343-351$

ISSN : 0246-8743

Référence électronique

André Meunier et Mary-Annick Morel, «Pas et point en français classique », Linx [En ligne], 5 | 1994, mis en ligne le 18 juillet 2012, consulté le 19 avril 2019. URL : http://journals.openedition.org/linx/1232 ;

DOI : 10.4000/linx.1232

Ce document a été généré automatiquement le 19 avril 2019.

Département de Sciences du langage, Université Paris Ouest 


\title{
Pas et point en français classique
}

\author{
André Meunier et Mary-Annick Morel
}

1 Les nombreuses études faites jusqu'à ce jour sur la valeur distinctive de pas et de point se limitent en fait à la syntaxe de la phrase. Nous voudrions, pour notre part, montrer qu'on peut préciser ce qui conditionne l'emploi respectif de point et de pas en prenant en considération leur distribution dans de grands ensembles discursifs et dans le cadre de l'interaction (dans le dialogue ou dans des textes de caractère dialogique), en prenant donc en compte les relations interpersonnelles.

\section{Hypothèse explicative de la valeur distinctive de pas et de point}

2 Notre hypothèse repose sur une étude exhaustive des emplois de pas et de point dans Les Femmes Savantes et Le Malade Imaginaire de Molière ${ }^{1}$. Nous allons donc tenter de préciser ce qui distingue ces deux adverbes sur le plan pragmatico-énonciatif, avant de passer en revue les contextes-types dans lesquels ils apparaissent. Nous rencontrerons chemin faisant la distinction opérée par Ducrot entre les trois valeurs de la négation (descriptive/ polémique/métalinguistique), mais notre objectif est de montrer que la distribution de ces deux adverbes est fondée sur un autre clivage ${ }^{2}$.

\subsection{Pas et point dans Molière: Phrases impératives et interrogatives}

3 Pas apparaît comme l'adverbe non marqué ( $58 \%$ des occurrences totales de la négation dans les deux pièces). On note toutefois une sorte de distribution complémentaire entre l'emploi majoritaire de point avec l'impératif et avec les verbes signifiant une modalité déontique - notamment il faut - $(72 \%)$ et l'emploi majoritaire de pas dans les phrases interrogatives ( $75 \%$ ). De plus, on ne relève, dans les deux pièces, aucun emploi de pas $d u$ tout, aucun emploi de la structure nominale pas de $N(100 \%$ de point en association avec $d u$ tout et avec de N). 
Argan : Tenez, mon frère, ne parlons point de cet homme-là davantage (MI III, 3)

Argan: Ne vous ai-je pas recommandé de me venir dire d'abord tout ce que vous voyez? (MI II, 8)

Philaminte : Je ne veux point d'obstacle aux désirs que je montre (FS II, 6)

Argan : Viens. N'aie point de peur (MI III, 14)

Henriette : Moi, ma sœur? point du tout (FS I, 2)

\subsection{Hypothèse explicative}

4 a) En recourant à l'interrogation, en suspendant donc l'assertion de l'énoncé, l'énonciateur impose à son interlocuteur de répondre et donc de prendre en charge dans sa réponse l'assertion suspendue. Il présuppose que l'interlocuteur est en mesure de le faire. Il s'appuie donc sur une sorte d'accord préalable, ou dit en d'autres termes sur la « consensualité co-énonciative ». Par co-énonciation il faut entendre la représentation que tout énonciateur se fait de ce que l'autre (son interlocuteur) pense. C'est l'emploi majoritaire de pas dans les phrases interrogatives qui permet, comme nous le verrons, d'expliquer sa valeur dans les autres types d'énoncés.

Argan : Eh! Ne diriez-vous pas que c'est effectivement Toinette?

Beralde : Il est vrai que la ressemblance est tout-à-fait grande.

Thomas Diafoirus : N'est-ce pas par le père qu'il convient de commencer?

Monsieur Diafoirus : Oui. (MI II, 5)

Toinette (qui surprend Argan) fait comme s'il l'avait appelée : Ne m'avez-vous pas

appelée? (MI III, 9)

b) L'impératif au contraire implique une représentation unilatérale de l'énonciateur, née d'un constat fait à partir de la réalité. Il n'est nullement fondé sur la co-énonciation. L'énoncé à l'impératif est simplement « adressé » à l'interlocuteur, qui reste libre de son attitude. Il traduit explicitement une rupture dans la co-énonciation et souligne l'attitude égocentrée de l'énonciateur. C'est cet emploi majoritaire de point dans les phrases à l'impératif qui permet, à notre avis, d'expliquer sa valeur énonciative propre.

6 On peut ainsi opposer les phrases qui traduisent une défense, une interdiction, un désaccord avec le co-énonciateur, notamment à l'impé-ratif 1ère personne du pluriel, qui requièrent l'emploi de point, à celles qui impliquent une exhortation, un conseil et qui recourent à pas. On peut aussi expliquer certains des rares emplois de pas avec l'impératif par la nature particulière des relations interpersonnelles, interdisant une attitude de rupture, comme on le voit clairement dans la façon dont les filles d'Argan, Angélique et Louison, s'adressent à leur père, ou dont Henriette réplique à Trissotin en présence de sa mère.

Henriette : Ne troublons point du ciel les justes règlements. (FS I, 1)

Philaminte : Ne faites point languir de si pressants désirs. (FS III, 1)

Angélique : De grâce, ne précipitez pas les choses. (MI II, 6)

Louison : Non, mon papa, ne le croyez pas. (MI II, 8)

Henriette : Tout beau, monsieur ! il n'est pas encore fait

Ne vous pressez pas tant. (FS III, 6)

c) Notre étude nous amène donc à nuancer l'interprétation de Littré concernant la valeur de point dans les phrases interrogatives. De fait, si les interrogations avec pas présentent bien un présupposé d'assertion positive, celles qui renferment point, en revanche, font basculer le présupposé du côté négatif. Point souligne, en effet, le fait que la réalité est contraire aux attentes de l'énonciateur et montre que c'est une représentation négative qui est en jeu au moment de l'énonciation. 
8 A son frère Chrysale qui se laisse gouverner par sa femme (cf. ses propos précédents « Elle me fait trembler dès qu'elle prend son ton.») et qui ne semble pas vouloir changer d'attitude, Ariste demande :

N'avez-vous point de honte avec votre mollesse? (MI II, 9)

Armande tente de ramener à elle Clitandre qui s'est détour-né d'elle, parce qu'il ne partage pas sa conception de l'amour :

Et vous ne goûtez point dans ses plus doux appas

Cette union des cœurs où les corps n'entrent pas? (FS IV, 2)

\section{Autres distributions liées à la valeur propre de pas et de point}

9 a) PAS apparaît chaque fois que l'énonciateur prend appui sur la co-énonciation, sur ce qu'il suppose de partagé par son interlocuteur. Il s'appuie le plus souvent sur ce qui a été dit au cours de l'échange et concerne plus particulièrement l'articulation des constituants de la phrase.

D'où son emploi naturel dans les subordonnées ouvertes par puisque ou par si. Avec puisque l'énonciateur implique qu'il s'agit de la reprise d'un fait présupposé connu et avec $s i$ il demande à son interlocuteur de prendre en considération un fait qui conditionne l'interprétation et la validité de la suite.

M. Purgon : Mais, puisque vous n'avez pas voulu guérir par mes mains... (MI III, 5)

Angélique : ...Si vous n'êtes pas favorable au penchant de mon cœur, si vous me refusez Cléante pour époux, je vous conjure, au moins, de ne me point forcer d'en épouser un autre. (MI III, 14)

Pas signifie le rejet de la relation prédicative.

Argan: On dira ce qu'on voudra, mais je vous dis que je veux qu'elle exécute la parole que j'ai donnée.

Toinette : Non, je suis sûre qu'elle ne le fera pas. (MI I, 5)

Angélique : Je pleure la mort de mon père...

Argan : N'aie point de peur, je ne suis pas mort. (MI III, 14)

Toinette : Je ne dis pas cela. (MI I, 4)

Pas est seul possible quand l'incidence de la négation est limitée à un constituant, notamment à une quantification ou une comparaison.

Béralde : ...et il y en a beaucoup parmi eux qui ne sont pas plus habiles que vous

(MI III, 14)

Toinette raillant: Ne parlez pas si haut (MI II, 2)

Pas opère un renvoi au contexte linguistique. C'est ce rôle anaphorique qui explique l'emploi systématique de pas dans le marqueur concessif cela n'empêche pas que, chaque fois qu'il y a reprise textuelle d'une séquence, ainsi que dans les propositions coordonnées par et.

Toinette: Il marche, dort, mange et boit tout comme les autres; mais cela

n'empêche pas qu'il ne soit fort malade. (MI II, 2)

J'ai cru jusques ici que c'était l'ignorance

Qui faisait les grands sots et non pas la science (FS 832)

Pas signifie un constat d'absence.

Cléante : Je n'ai pas une voix à chanter. (MI II, 5)

Clitandre : Eh Madame, à quoi bon un pareil embarras

Et pourquoi voulez-vous penser ce qui n'est pas? (MI II, 5)

Bélise : Non, non, je vous entends, vous ignorez l'histoire

Et l'affaire n'est pas ce que vous pouvez croire. (MI II, 3) 
10 b) POINT s'emploie lorsqu'il y a rupture dans la co-énonciation et rejet en bloc de l'attitude ou de l'argument de l'autre.

Point peut, à la différence de pas, constituer un énoncé à part entière (souvent renforcé par $d u$ tout): il est alors comme non, une prophrase anaphorique qui a une incidence globale.

Trissotin : Peut-être que mes vers importunent Madame?

Henriette : Point : je n'écoute pas. (FS III, 2)

Monsieur Purgon: Puisque vous vous êtes déclaré rebelle aux remèdes que je vous

ordonnais...

Argan : Hé ! point du tout (MI III, 5)

11 Point souligne un égocentrage fort de l'énonciateur. Il apparaît souvent dans des contextes riches en déictiques et fortement modalisés, où l'énonciateur se donne explicitement comme étant le support de la modalité. Il est dominant avec les verbes de volonté et de sentiment, qui rejoignent la modalité déontique examinée ci-dessus avec l'impératif. Il est à noter que les modaux de type épistémique s'accompagnent plus souvent de pas. L'explication est à chercher dans leur moindre charge affective que les déontiques ou les évaluatifs. D'où une présence importante de pas avec l'impersonnel (moins pris en charge que l'énoncé personnel).

Clitandre : Ceci n'est point du tout un trait d'esprit, madame, (FS I, 4)

Toinette : Je ne veux point qu'elle épouse votre Thomas Diafoirus. (MI I, 2)

Henriette : Et le mépris du bien et des grandeurs frivoles

Ne doit point éclater dans vos seules paroles. (FS V,1)

Clitandre : Et j'ose maintenant vous conjurer, Madame,

De ne vouloir tenter nul effort sur ma flamme,

De ne point essayer à rappeler un cœur... (FS 1,2)

Chrysale : Rien, et je suis bien aise

De n'avoir point parlé pour ne m'engager pas. (FS II, 9)

Chrysale : Ma foi, je ne sais pas. (FS II, 6)

Bélise : Et je ne savais pas que j'eusse des chimères. (FS II, 3)

\section{Rôle respectif de pas et de point dans l'argumentation}

12 a) Point signifie le refus et apparaît avec la valeur d'une négation polémique (incluant la négation métalinguistique). Dans le dialogue serré et argumenté, point domine, surtout dans une stratégie de contre-argumentation, où il s'agit essentiellement de réfuter. Pas apparaît, au contraire, lorsqu'il y a désir de convaincre, d'emporter l'adhésion.

Armande : Ainsi donc à leurs vœux vous me sacrifiez?

Philaminte : Ce ne sera point vous que je leur sacrifie (FS V, 4)

Toinette : Je pensais, Madame, qu'il fallût pleurer.

Bélise : Va, va, cela n'en vaut pas la peine. (MI III, 16)

Dans l'échange entre Martine et Chrysale (FS V, 3), l'emploi quasi-systématique de point qu'on relève dans les répliques de Martine n'est pas tant à mettre au compte d'une particularité dialectale, mais bien plus de son insolence vis-à-vis de son maître (énoncés de type déontique ou prise de position personnelle marquée). Ainsi entend-on :

Ce n'est point à la femme à prescrire.

La poule ne doit point chanter devant le coq.

Je ne l'aimerais point s'il faisait le Jocrisse.

L'esprit n'est point du tout ce qu'il faut en ménage. 
Et je veux, si jamais on engage ma foi,

Un mari qui n'ait point d'autre livre que moi. face à

Il lui faut un mari, non pas un pédagogue.

Elle n'a pas besoin de monsieur Trissotin. (FS V, 3)

Dans un dialogue serré où les deux interlocuteurs ont des positions irréductibles, les deux marqueurs négatifs alternent dans un jeu subtil, ainsi dans MI I, 5, lignes 122-173:

1.123- et elle n'est point faite pour être Madame Diafoirus (réfutation de Toinette)

1.126- Eh ! fi ! ne dites pas cela. (conseil, exhortation de T.)

1.127- Comment ! que je ne dise pas cela? (reprise textuelle par A.)

1.141- Vous ne la mettrez point dans un couvent. (valeur déontique de la part de T.)

1.144- Je ne la mettrai point dans un couvent? (reprise textuelle en discours rapporté par A.)

1.148- Voici qui est plaisant ! Je ne mettrai pas ma fille dans un couvent, si je veux?

(reprise en discours indirect libre des propos de Toinette: Argan n'est pas

l'énonciateur et l'énoncé perd de sa subjectivité)

1.154-155- Vous vous moquez (T.)

Je ne me moque point (réfutation égocentrée d'A.)

1.156-157- La tendresse paternelle vous prendra ( $\mathrm{T}$.)

Elle ne me prendra point. (idem A.)

1.164-165- Bagatelles (T.)

Il ne faut point dire : Bagatelles (valeur déontique, réfutation d'A.)

b) Revenant aux exemples proposés dans le Littré et dans le Robert, on a confirmation que point apparaît de manière privilégiée dans des énoncés où la négation est accompagnée d'un effet de contraste (cf. Voltaire), de contre-argumentation (cf. le renversement argumentatif dans la phrase de Pascal), caractéristique d'une énonciation dialogale (cf. Corneille) ou dialogique (cf. Gide).

Je suis près de ma salle à manger où je ne mange point ; je vois mon jardin où je ne me promène point ; j'ai autour de moi des sociétés dont je ne jouis point. (Voltaire) Pour défendre le bien public, plusieurs le font, mais pour la religion, point. (Pascal)

Chimène : Va, je ne te hais point. (Corneille)

Je ne vous réponds pas des volontés d'un père

Mais je ne serai point à d'autre qu'à Valère. (Molière, Tartuffe II, 5)

L'amour peut être aveugle ; l'amitié point : elle se doit de ne point l'être. (Gide)

Mais il avait beau supputer toutes les chances, il n'espérait point retirer cent francs de son effort. Il ne parvenait pas à estimer sa valeur commerçante à plus de cinquante francs. (Mac Orlan, cité par Wagner et Pinchon, p. 421)

Dans l'exemple de Mac Orlan, la structure concessive renforce le renversement argumentatif marqué par la négation point. L'apparition de pas dans la phrase suivante nous ramène au constat d'absence. On trouve du reste le même effet de contraste argumentatif dans l'alternance de point et de pas, chez Molière. De la même manière, l'emploi de point succédant à non marque un renforcement de l'attitude polémique.

Toinette : Mais votre fille doit épouser un mari pour elle et n'étant point malade, il

n'est pas nécessaire de lui donner un médecin. (MI I, 5)

Louison : Là, là mon papa, ne pleurez point tant; je ne suis pas morte tout-à-fait.

(MI II, 8)

Angélique : Montrez-vous généreux.

G. Dandin : Non.

Angélique : De grâce.

G. Dandin : Point. (Georges Dandin III, 8, cité par Littré) 


\section{Rôle respectif de pas et de point dans le récit}

18 a) Dans les monologues longs ou dans les récits, on attendrait une dominance de pas, signifiant un constat d'absence objectif (cf. ci-dessous «ma sonnette ne fait pas assez de bruit»). Mais des ruptures sont toujours possibles : point signale alors le décalage entre l'attente et ce qui est constaté dans la réalité (cf. « et ne suis point servi ») ou la rupture avec la pensée de l'autre (cf. «ne sont point de mon goût »).

Argan : J'ai beau dire on me laisse toujours seul. Il n'y a pas moyen de les arrêter ici.

(Il agite une sonnette pour faire venir ses gens). Ils n'entendent point, et ma

sonnette ne fait pas assez de bruit. (MI I, 2)

Chrysale : Et l'on sait tout chez moi, hors ce qu'il faut savoir.

On y sait comme vent, lune, étoile polaire

Vénus, Saturne et Mars dont je n'ai point affaire...

Et j'ai des serviteurs et ne suis point servi.

Une pauvre servante au moins m'était restée

Qui de ce mauvais air n'était point infectée (FS II, 7)

Clitandre (à Henriette) : Mon cœur n'a jamais pu, tant il est né sincère,

Même dans votre sœur flatter leur caractère,

Et les femmes docteurs ne sont point de mon goût.

Je consens qu'une femme ait des clartés de tout,

Mais je ne lui veux point la passion choquante

De se rendre savante afin d'être savante. (FS I, 3)

b) Un rapide sondage effectué dans l'œuvre de Robert Challe Les illustres Françaises nous a montré que, dans ce type de roman où le narrateur principal est mis en scène à la première personne, l'emploi de point signale une rupture dans la narration en cours. Il marque qu'il y a un changement de point de vue, qu'il s'agit d'un commentaire du narrateur lui-même, et non du personnage concerné par le récit. Il en va de même du reste dans les dialogues entre les personnages. La rupture ainsi produite pas point est du reste corroborée par la co-occurrence avec des marques fortes d'égocentrage (cf. ici et la focalisation par le présentatif, p.197), par le passage à un autre plan énonciatif (cf. l'impératif et l'apostrophe dans allez, Monsieur, p. 193) ou par un commentaire du narrateur (cf. avec dépit, p. 193) :

Pour revenir à Mademoiselle Grandet, ce fut ainsi que je rompis avec elle, et je vous laisse à penser si elle n'est pas en droit de me regarder comme un fourbe et comme un scélérat?

Je n'interrompis point Jussy en cet endroit de sa narration, poursuivit Des Frans, en s'interrompant soi-même, et parlant à Madame de Mongey. Ce ne fut point ici que je lui dis que j'avais l'honneur de vous connaître (p. 196-197)

- [...] Non, Mademoiselle, poursuivis-je, vous méritez un tout autre parti que moi. Une meilleure fortune vous attend,

et je ne dois pas, non seulement vous laisser borner vos espérances, mais même déchoir de l'état où vous êtes née. [...] - Je n'attendais pas un pareil conseil de votre part, me dit-elle;[...] Je vois bien que vous aimez Mademoiselle Grandet, puisque vous recevez si mal mes offres; allez, Monsieur, continua-t-elle avec dépit, je ne veux point retarder votre bonheur. (p. 193)

\section{Conclusion}

Notre investigation sur les deux pièces de Molière et sur un certain nombre d'autres textes classiques montre que la distribution de pas et de point peut être en grande partie 
expliquée par des phénomènes d'ordre énonciatif. Pas sert à mettre en avant la consensualité co-énonciative, l'accord de la représentation de l'énonciateur avec celle qu'il prête à son interlocuteur. Point, au contraire, traduit une rupture par rapport à un accord préalable et par suite souligne la position fortement égocentrée de l'énonciateur. Ainsi s'expliquent à nos yeux les effets produits par l'emploi de l'un ou de l'autre de ces deux marqueurs négatifs dans un échange dialogué, dans une argumentation, et même dans un récit. Notons pour finir que, par suite de la diminution progressive des emplois de point, c'est pas qui a "récupéré » au fur et à mesure ses valeurs. Nous reconnaissons qu'il devient très difficile, à partir de la fin du XVIIIème siècle, d'appliquer notre hypothèse explicative, sauf s'il s'agit d'écrivains qui, tel Gide, sont très marqués par la langue classique.

\section{NOTES}

1. Editions consultées : Le malade imaginaire, Classique Hatier, œuvres et thèmes, 1981 ; Les femmes savantes, Classiques Larousse.

2. Nous avons toutefois observé que certains emplois peuvent s'expliquer par la nature nominale de l'énoncé (MI I, 1), ou encore par des raisons rythmiques ou un effet rhétorique (cf. de ce pas/ ne...pas) :

Argan : Drelin, drelin, drelin, point d'affaire. (MI I, 1)

Philaminte : Henriette et monsieur seront joints de ce pas ;

Je l'ai dit, je le veux : ne me répliquez pas. (FS V, 3)

\section{RÉSUMÉS}

La distribution des forclusifs pas et point, observée dans des textes classiques (notamment dans le théâtre de Molière) ne constitue pas, comme il est dit généralement, un cas de variation libre (ou dialectale), mais peut être en grande partie expliquée par des phénomènes d'ordre énonciatif : pas signale une consensualité co-énonciative entre les représentations de l'énonciateur et celles qu'il prête à son co-énonciateur ; point au contraire souligne une discordance (une rupture) par rapport à cette consensualité. La généralisation de pas au fil de l'histoire a progressivement réduit cette opposition, perceptible cependant encore chez les auteurs contemporains archaïsants.

The distribution of the "Forclusives" pas and point, which can be observed in classical texts (specillay Moliere's plays), is not, as generally claimed, a case of dialectical variation, but it can be largely explained by phenomena related to enunciation. By using pas the "enunciator" reveals a family likeness or an agreement between his own thoughts and those of the "co-enunciator". On the contrary, point shows a discrepancy. But, as a result of pas becoming predominent in 
French, the contrast has gradually been reduced, except among contemporary writers who are fond of out-dated language. 